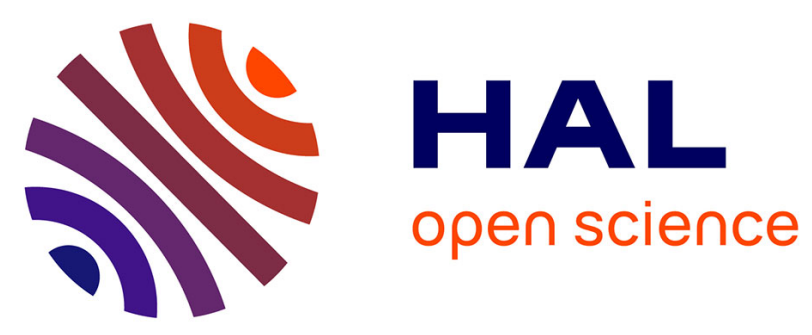

\title{
Production equilibria
}

Charalambos D. Aliprantis, Monique Florenzano, Rabee Tourky

\section{To cite this version:}

Charalambos D. Aliprantis, Monique Florenzano, Rabee Tourky. Production equilibria. Journal of Mathematical Economics, 2006, 42 (4-5), pp.406-421. 10.1016/j.jmateco.2006.04.006 . halshs00092809

\section{HAL Id: halshs-00092809 \\ https://shs.hal.science/halshs-00092809}

Submitted on 12 Sep 2006

HAL is a multi-disciplinary open access archive for the deposit and dissemination of scientific research documents, whether they are published or not. The documents may come from teaching and research institutions in France or abroad, or from public or private research centers.
L'archive ouverte pluridisciplinaire HAL, est destinée au dépôt et à la diffusion de documents scientifiques de niveau recherche, publiés ou non, émanant des établissements d'enseignement et de recherche français ou étrangers, des laboratoires publics ou privés. 


\title{
PRODUCTION EQUILIBRIA
}

\author{
CHARALAMBOS D. ALIPRANTIS ${ }^{1}$, MONIQUE FLORENZANO $^{2}$, AND RABEE TOURKY $^{1}$
}

\begin{abstract}
${ }^{1}$ Department of Economics, Krannert School of Management, Purdue University, 403 West State Street, W. Lafayette, IN 47907-2056, USA; aliprantis@mgmt.purdue.edu; rtourky@purdue.edu

2 Centre d'Economie de la Sorbonne, UMR 8174 CNRS-Université Paris 1, 106-112 boulevard de l'Hôpital, 75647 Paris Cedex 13, FRANCE; monique.florenzano@univ-paris1.fr
\end{abstract}

\begin{abstract}
This paper studies production economies in a commodity space that is an ordered locally convex space. We establish a general theorem on the existence of equilibrium without requiring that the commodity space or its dual be a vector lattice. Such commodity spaces arise in models of portfolio trading where the absence of some option usually means the absence of a vector lattice structure. The conditions on preferences and production sets are at least as general as those imposed in the literature dealing with vector lattice commodity spaces. The main assumption on the order structure is that the Riesz-Kantorovich functionals satisfy a uniform properness condition that can be formulated in terms of a duality property that is readily checked. This condition is satisfied in a vector lattice commodity space but there are many examples of other commodity spaces that satisfy the condition, which are not vector lattices, have no order unit, and do not have either the decomposition property or its approximate versions.
\end{abstract}

JEL classification: C61; C62; D20; D46; D51

Keywords: Production economies; Equilibrium; Edgeworth equilibrium; Properness; RieszKantorovich functional; Sup-convolution

\section{INTRODUCTION}

This paper is part of a series of papers studying Walrasian equilibria in ordered vector spaces that need not be vector lattices. The other papers in this series are Aliprantis, Florenzano, and Tourky (2004b, 2005); Aliprantis, Florenzano, Martins-da-Rocha, and Tourky (2004a). The paper extends to production economies results obtained in Aliprantis, Florenzano, and Tourky (2004b) for exchange economies.

In our papers Aliprantis, Florenzano, and Tourky (2004b, 2005) we established necessary and sufficient conditions for the existences of equilibrium in exchange economies with an ordered topological vector commodity space and preferences that satisfy the properness assumption. We were motivated by models in finance with commodity spaces that are not vector lattices. In models of

A first version of this paper has been presented at the 11th Conference on Real Analysis and Measure Theory in Ischia (Italy, 2004). This version was presented at the Debreu Memorial Conference in Berkeley (USA, 2005). The paper has benefitted of the comments of diverse audiences. We thank an anonymous referee for a number of important questions whose solutions have improved the paper. We also thank V. Filippe da Rocha for useful suggestions. The research of C. D. Aliprantis is supported by the NSF Grants EIA-0075506, SES-0128039, and DMS-0437210. The research of R. Tourky was funded by the Australian Research Council Grant A00103450. 
portfolio trading we have a pair of function spaces $L$ and $X$, where $L$ is the portfolio space and $X$ is the space of contingent claims, together with a positive linear operator $A: L \rightarrow X$ that pulls back the order intervals of $A(L)$ to closed and bounded subsets of $L$. The portfolio dominance ordering, a notion introduced in Aliprantis, Brown, and Werner (2000), is the only ordering on the portfolio space which is relevant for purposes of economic analysis of arbitrage free security prices. However, if the portfolio space is reordered by the portfolio dominance ordering, then it is seldom a vector lattice and the existence of equilibrium cannot be deduced from theorems that require lattice properties on the commodity space. Our papers Aliprantis, Florenzano, and Tourky (2004b, 2005) solve this problem for exchange economies in the sense that they provide necessary and sufficient conditions on the order structure of the commodity (portfolio) space, and therefore in terms of the assets structure $A: L \rightarrow X$. In particular, if our conditions do not hold, then one can construct an economy satisfying the usual assumptions on preferences that has no linear price equilibrium. When our conditions hold the usual assumptions guarantee the existence of equilibrium with linear prices. If there is no linear price equilibrium, then one must invoke the theory of value with personalized prices developed in Aliprantis, Tourky, and Yannelis (2001).

In this paper, we study the existence of general equilibrium for production economies with an ordered locally convex commodity space. Our assumptions are quite general. Preferences are not ordered, we do not assume any monotonicity of preferences or free disposal in production. We also assume general properness assumptions on preference and production sets. The modern literature establishing the existence of equilibrium with general assumptions on preferences and production sets is divided into two groups. The first considers the setting of Mas-Colell (1986a), where the commodity space is a locally solid topological vector lattice. The main papers in this group include Aliprantis, Brown, and Burkinshaw (1987b); Zame (1987). The second group follows MasColell and Richard (1991) and assumes that the commodity space is a locally convex vector lattice whose topological dual is a vector sublattice of the order dual. That is, they drop the local solidness assumption of the earlier literature. The main contributions in this setting include Richard (1989); Tourky (1999); Florenzano and Marakulin (2001). In both literatures the lattice structures of the commodity-price duality are used in non-trivial ways.

The novelty of this paper is to replace the lattice properties of the commodity-price duality by a condition on the order structure of the commodity space. As with our the other papers in the series, the present paper uses the duality property of the Riesz-Kantorovich functionals whose study was initiated in Aliprantis, Tourky, and Yannelis (2000, 2001); Aliprantis and Tourky (2002) and was further motivated by the three assets portfolio trading example of Aliprantis, Monteiro, and Tourky (2004c).

The paper is organized as follows. In section 2, we recall the notion of the Riesz-Kantorovich functional associated with a finite list of (linear) functionals and the concept of sup-convolution in optimization. In section 3, we define the model and set out our assumptions. Section 4 is devoted to the decentralization of Edgeworth equilibria with linear prices and thus establishes the existence of equilibrium.

\section{Preliminaries}

For details regarding Riesz spaces that are not explained below we refer the reader to Aliprantis and Burkinshaw $(1985,2003)$. This paper will utilize the notion of the Riesz-Kantorovich functional studied in Aliprantis, Tourky, and Yannelis (2000); Aliprantis and Tourky (2002) and used extensively in Aliprantis, Tourky, and Yannelis (2001); Aliprantis, Florenzano, and Tourky (2004b, 
2005). We shall briefly introduce this formula here and refer the reader to Aliprantis and Tourky (2002) for a more complete discussion.

Let $L$ be an ordered vector space. Recall that the decomposition property states that if $x$, $y_{1}, y_{2} \in L_{+}$satisfy $0 \leq x \leq y_{1}+y_{2}$, then there exist vectors $x_{1}$ and $x_{2}$ such that $0 \leq x_{1} \leq y_{1}$, $0 \leq x_{2} \leq y_{2}$ and $x=x_{1}+x_{2}$. The order dual $L^{\sim}$ is the vector space consisting of all linear functionals on $L$ which map order intervals of $L$ to order bounded subsets of $\mathbb{R}$, ordered by the relation $f \geq g$ whenever $f(x) \geq g(x)$ for all $x \in L_{+}$.

We start with the following classical result from the theory of partially ordered vector spaces due to F. Riesz and L. V. Kantorovich.

Theorem 2.1 (Riesz-Kantorovich). If $L$ is an ordered vector space with a generating cone and the decomposition property, then the order dual $L^{\sim}$ is a Riesz space and for each $f, g \in L^{\sim}$ and $x \in L_{+}$its lattice operations are given by:

$$
\begin{aligned}
& {[f \vee g](x)=\sup \left\{f(y)+g(z): y, z \in L_{+} \text {and } y+z=x\right\}, \text { and }} \\
& {[f \wedge g](x)=\inf \left\{f(y)+g(z): y, z \in L_{+} \text {and } y+z=x\right\}}
\end{aligned}
$$

In particular, if $L$ has the decomposition property, then for any finite collection of linear functionals $f_{1}, f_{2}, \ldots, f_{m} \in L^{\sim}$ their supremum in $L^{\sim}$ at each $x \in L_{+}$is given by

$$
\left[\bigvee_{i=1}^{m} f_{i}\right](x)=\sup \left\{\sum_{i=1}^{m} f_{i}\left(x_{i}\right): x_{i} \in L_{+} \text {for each } i \text { and } \sum_{i=1}^{m} x_{i}=x\right\} .
$$

For any positive integer $m$ and $x \in L_{+}$define

$$
\mathcal{A}_{x}^{m}=\left\{\left(x_{1}, \ldots, x_{m}\right) \in L_{+}^{m}: \sum_{i=1}^{m} x_{i}=x\right\} .
$$

The formula $(\star)$ that gives the supremum of the order bounded linear functionals $f_{1}, \ldots, f_{m}$ is called the Riesz-Kantorovich formula of these functionals. The useful observation here is that if each $f_{i}$ is an arbitrary function from $L_{+}$to $(-\infty, \infty]$, then the right-hand side of $(\star)$ still defines an extended real number for each $x \in L_{+}$. That is, the formula appearing in $(\star)$ defines a function from $L_{+}$to $(-\infty, \infty]$ called the Riesz-Kantorovich functional of the $m$-tuple of functions $f=\left(f_{1}, \ldots, f_{m}\right)$ and denoted $\mathcal{R}_{f}$. In other words, $\mathcal{R}_{f}: L_{+} \rightarrow(-\infty, \infty]$ is defined for each $x \in L_{+}$ by

$$
\mathcal{R}_{f}(x):=\sup \left\{\sum_{i=1}^{m} f_{i}\left(x_{i}\right):\left(x_{1}, \ldots, x_{m}\right) \in \mathcal{A}_{x}^{m}\right\} .
$$

If each $f_{i}$ is a function that carries order intervals to bounded from above subsets of $\mathbb{R}$, then the Riesz-Kantorovich functional is real-valued. Moreover, if each $f_{i}$ is super-additive and positively homogeneous, then the Riesz-Kantorovich functional $\mathcal{R}_{f}$ is likewise super-additive and positively homogeneous; in particular, it is a concave function.

Let us now associate with each $f_{i}: L_{+} \rightarrow(-\infty, \infty)$ the function $\widehat{f}_{i}: L \rightarrow[-\infty, \infty)$ defined by

$$
\widehat{f_{i}}(x):=\left\{\begin{array}{cc}
f_{i}(x) & \text { if } x \in L_{+}, \\
-\infty & \text { otherwise }
\end{array}\right.
$$


If each $f_{i}$ is finite-valued, it is easy to see that for $x \in L_{+}, \mathcal{R}_{f}(x)$ is given by the value at $x$ of the sup-convolution ${ }^{1}$ of functions $\widehat{f}_{i}$ defined by

$$
\left[\nabla_{i=1}^{m} \widehat{f}_{i}\right](x):=\sup \left\{\sum_{i=1}^{m} \widehat{f}_{i}\left(x_{i}\right): \sum_{i=1}^{m} x_{i}=x\right\} .
$$

Definition 2.2. We will say that $\mathcal{R}_{f}$ is exact at $x$ with respect to a vector $\left(x_{1}, \ldots, x_{m}\right) \in \mathcal{A}_{x}^{m}$ if

$$
\mathcal{R}_{f}(x)=\left[\nabla_{i=1}^{m} \widehat{f}_{i}\right](x)=\sum_{i=1}^{m} \widehat{f}_{i}\left(x_{i}\right)=\sum_{i=1}^{m} f_{i}\left(x_{i}\right) .
$$

Let $\left\langle X, X^{\prime}\right\rangle$ be a dual system and let $f \in \overline{\mathbb{R}}^{X}$. Recall that a vector $y^{\prime} \in X^{\prime}$ is called a supergradient of $f$ at $x$ if $f(x)$ is finite and $f(y)-f(x) \leq\left\langle y-x, y^{\prime}\right\rangle$ for all $y \in X$. The (possibly empty) set of all supergradients of $f$ at $x$ is called the superdifferential at $x$ of the function $f$ and denoted $\partial f(x)$.

The following standard result, see for example (Laurent, 1972, Proposition 6.6.4), will be used in our work.

Theorem 2.3 (Moreau, 1967). Assume that $\left\langle X, X^{\prime}\right\rangle$ is an arbitrary dual system. For each $i=$ $1, \ldots, m$, let $g_{i}: X \rightarrow[-\infty, \infty]$ be a non identically equal to $-\infty$ function. If the sup-convolution $\nabla_{i=1}^{m} g_{i}$ is exact at $x$ with respect to some $\left(x_{1}, \ldots, x_{m}\right) \in X^{m}$ that satisfies $x=\sum_{i=1}^{m} x_{i}$, then

$$
\partial\left[\nabla_{i=1}^{m} g_{i}\right](x)=\bigcap_{i=1}^{m} \partial g_{i}\left(x_{i}\right)
$$

\section{The ECONOMIC MODEL}

In what follows, if $L$ is an ordered linear space, then for the sake of notational convenience, $L^{+}$or $L_{+}$will denote the positive cone of $L$. The commodity space of our model is an ordered vector space $L$ equipped with a Hausdorff locally convex topology $\tau$ such that:

(A1) The positive cone $L_{+}$of $L$ is generating (i.e., $L=L_{+}-L_{+}$) and $\tau$-closed.

(A2) The order intervals of $L$ are $\tau$-bounded.

The topological dual of $(L, \tau)$ (i.e., the vector space of all $\tau$-continuous linear functionals on $L$ ) will be denoted $L^{\prime}$. The algebraic dual of $L$ (i.e., the vector space of all linear functionals on $L$ ) is denoted $L^{*}$. The order dual of $L$ (i.e., the vector space of all order-bounded linear functionals on $L$ ) is denoted $L^{\sim}$. Since every order interval of $L$ is $\tau$-bounded, it follows that $L^{\prime} \subseteq L^{\sim} \subseteq L^{*}$.

\footnotetext{
${ }^{1}$ Let $\overline{\mathbb{R}}=\mathbb{R} \cup\{-\infty, \infty\}$ denote the set of extended reals. Using the convention $+\infty+(-\infty)=-\infty+(+\infty)=-\infty$, the addition in $\mathbb{R}$ can be extended to $\overline{\mathbb{R}}$. Then for $f \in \overline{\mathbb{R}}^{L}$ and $g \in \overline{\mathbb{R}}^{L}$, the formula $[f \nabla g](x)=\sup \{f(y)+g(z): y, z \in$ $L$ and $y+z=x\}$ defines an extended real-valued function $f \nabla g$ called the sup-convolution of $f$ and $g$. The expression $f \nabla g$ is also called by Rockafellar and Wets (1998) the hypo-addition of functions $f$ and $g$, because if hypo $f$ denotes the hypograph of $f$, one has hypo $(f \nabla g)=$ hypo $f+$ hypo $g$, as long as the supremum defining $[f \nabla g](x)$ is attained when finite.

More generally, for any finite collection of functions $g_{1}, g_{2}, \ldots, g_{m} \in \overline{\mathbb{R}}^{L}$, the formula $\nabla_{i=1}^{m} g_{i}(x)=$ $\sup \left\{\sum_{i=1}^{m} g_{i}\left(x_{i}\right): \sum_{i=1}^{m} x_{i}=x\right\}$ defines the sup-convolution of functions $g_{i}$. The sup-convolution $\nabla_{i=1}^{m} g_{i}$ is said to be exact at $x=\sum_{i=1}^{m} x_{i}$ if $\nabla_{i=1}^{m} g_{i}(x)=\sum_{i=1}^{m} g_{i}\left(x_{i}\right)$.
} 
Given the commodity space $L$, a private ownership production economy is an ordered tuple

$$
\mathcal{E}=\left(\left(X_{i}, P_{i}, \omega_{i}\right)_{i \in I},\left(Y_{j}\right)_{j \in J},\left(\theta_{i j}\right)_{i \in I, j \in J}\right)
$$

where $I=\{1, \ldots, m\}$ is a finite set of $m(\geq 2)$ consumers, $J=\{1, \ldots, n\}$ is a finite set of $n(\geq 1)$ producers. Each consumer $i$ is characterized by a non-empty consumption set $X_{i} \subseteq L$, an initial endowment $\omega_{i} \in X_{i}$ and an irreflexive preference correspondence $P_{i}: X_{i} \rightarrow X_{i}$, i.e., $x_{i} \notin P_{i}\left(x_{i}\right)$ for each $x_{i} \in X_{i}$. Each producer $j$ is characterized by a non-empty production set $Y_{j} \subseteq L$. For every producer and each consumer, the firm shares $0 \leq \theta_{i j} \leq 1$ classically represent a contractual claim of consumer $i$ to the profit of producer $j$ and $\sum_{i \in I} \theta_{i j}=1$ for each $j \in J$. In a core and Edgeworth equilibrium approach, the relative shares $\theta_{i j}$ reflect consumers' stockholdings that represent proprietorships of production possibilities and $\theta_{i j} Y_{j}$ is the portion of the $j$ producer's technology set at $i$ 's disposal. is,

Let $\omega=\sum_{i \in I} \omega_{i}$ be the total endowment, and let $\mathcal{A}_{\omega}$ be the set of all allocations of $\mathcal{E}$, that

$$
\mathcal{A}_{\omega}=\left\{(x, y)=\left(\left(x_{i}\right)_{i \in I},\left(y_{j}\right)_{j \in J}\right) \in \prod_{i \in I} X_{i} \times \prod_{j \in J} Y_{j}: \sum_{i \in I} x_{i}=\omega+\sum_{j \in J} y_{j}\right\} .
$$

The set $X_{\omega}$, the projection of $\mathcal{A}_{\omega}$ on $\prod_{i \in I} X_{i}{ }^{2}$ is the set of all consumption allocations.

We recall the following standard notions of equilibria for an economy $\mathcal{E}$.

Definition 3.1. A 3-tuple $(\bar{x}, \bar{y}, \bar{p})$ consisting of an allocation $(\bar{x}, \bar{y})$ and a non-zero linear functional $\bar{p}$ is said to be:

(1) a quasi-equilibrium, if

(a) for every $i \in I$ we have $\bar{p}\left(\bar{x}_{i}\right)=\bar{p}\left(\omega_{i}\right)+\sum_{j \in J} \theta_{i j} \bar{p}\left(\bar{y}_{j}\right)$ and $x_{i} \in P_{i}\left(\bar{x}_{i}\right)$ implies $\bar{p}\left(x_{i}\right) \geq \bar{p}\left(\bar{x}_{i}\right)$, and

(b) for every $j \in J$ and every $y_{j} \in Y_{j}$ we have $\bar{p}\left(y_{j}\right) \leq \bar{p}\left(\bar{y}_{j}\right)$;

(2) an equilibrium, if it is a quasi-equilibrium and if $x_{i} \in P_{i}\left(\bar{x}_{i}\right)$ implies $\bar{p}\left(x_{i}\right)>\bar{p}\left(\bar{x}_{i}\right)$.

We emphasize that the notion of quasi-equilibrium in the infinite dimensional setting is vacuous without an additional restriction.

Definition 3.2. A quasi-equilibrium $(\bar{x}, \bar{y}, \bar{p})$ is said to be non-trivial if for some $i \in I$ we have

$$
\inf \left\{\bar{p}\left(z_{i}\right): z_{i} \in X_{i}\right\}<\bar{p}\left(\bar{x}_{i}\right) \text {. }
$$

In this paper, we will be interested only in non-trivial quasi-equilibria. If $(\bar{x}, \bar{y}, \bar{p})$ is some trivial quasi-equilibrium, then for every feasible allocation $(x, y)$, the pair $((x, y), \bar{p})$ is also a quasi-equilibrium. On the other hand, if the quasi-equilibrium $(\bar{x}, \bar{y}, \bar{p})$ is non-trivial, then it is well-known that, under some continuity condition on preferences or concavity for utility functions and some weak irreducibility assumption on the economy, the quasi-equilibrium $(\bar{x}, \bar{y}, \bar{p})$ is actually an equilibrium.

The following notions of optimality are also standard.

Definition 3.3. A consumption allocation $\bar{x} \in X_{\omega}$ is said to be:

(1) weakly Pareto optimal, if there is no consumption allocation $x \in X_{\omega}$ satisfying $x_{i} \in$ $P_{i}\left(\bar{x}_{i}\right)$ for each $i \in I$,

${ }^{2}$ That is, $X_{\omega}=\left\{x=\left(x_{i}\right)_{i \in I} \in \prod_{i \in I} X_{i}: \quad \sum_{i \in I} x_{i}=\omega+\sum_{j \in J} y_{j}\right.$ for some $\left.\left(y_{j}\right)_{j \in J} \in \prod_{j \in J} Y_{j}\right\}$. 
(2) a core allocation, if it cannot be blocked by any coalition in the sense that there is no coalition $S \subseteq I$ and some $x \in \prod_{i \in S} X_{i}$ such that:

(a) $\sum_{i \in S} x_{i} \in \sum_{i \in S} \omega_{i}+\sum_{i \in S} \sum_{j \in J} \theta_{i j} Y_{j}$, and

(b) $x_{i} \in P_{i}\left(\bar{x}_{i}\right)$ for all $i \in S$,

(3) an Edgeworth equilibrium, if it belongs to the core of every $r$-fold replica of $\mathcal{E},^{3}$

(4) a fuzzy core allocation, if there exist no $\tau=\left(\tau_{i}\right)_{i \in I} \in[0,1]^{I} \backslash\{0\}$ and $x \in \prod_{i \in I} X_{i}$ such that:

(a) $\sum_{i \in I} \tau_{i} x_{i} \in \sum_{i \in I} \tau_{i} \omega_{i}+\sum_{i \in I} \tau_{i} \sum_{j \in J} \theta_{i j} Y_{j}$, and

(b) $x_{i} \in P_{i}\left(\bar{x}_{i}\right)$ for all $i \in I$ with $\tau_{i}>0$.

From now on, we impose on $\mathcal{E}$ the following conditions.

Standard assumptions and $\omega$-properness: That is,

(1) For each consumer $i$, the consumption set $X_{i}$ is convex, and $\omega=\sum_{i \in I} \omega_{i}>0$.

(2) For each $i$ and every weakly Pareto optimal consumption allocation $x=\left(x_{i}\right)_{i \in I}$, $x_{i} \in \operatorname{cl} P_{i}\left(x_{i}\right), P_{i}\left(x_{i}\right)$ is open in $X_{i}$ for some linear topology on $L$ (or is induced by a concave utility function) and is $\omega$-proper at $x_{i}$ in the following sense adapted from Tourky (1998): There exist in $L$ a convex set $\widehat{P}_{i}\left(x_{i}\right)$ and a convex set $Z_{i}\left(x_{i}\right)$ such that

(a) the vector $x_{i}+\omega$ is a $\tau$-interior point of $\widehat{P}_{i}\left(x_{i}\right)$,

(b) $\widehat{P}_{i}\left(x_{i}\right) \cap Z_{i}\left(x_{i}\right)=P_{i}\left(x_{i}\right)$,

(c) $x_{i}, 0, \omega_{i} \in Z_{i}\left(x_{i}\right), Z_{i}\left(x_{i}\right)+L_{+} \subseteq Z_{i}\left(x_{i}\right)$, and

(d) for every $u>0$ and every couple $\left\{z_{i}, z_{i}^{\prime}\right\}$ of elements of $Z_{i}\left(x_{i}\right)$, if $-u \leq z_{i}$ and $-u \leq z_{i}^{\prime}$, then there exists $z \in Z_{i}\left(x_{i}\right)$ such that $-u \leq z \leq z_{i}$ and $-u \leq z \leq z_{i}^{\prime}$.

(3) For each producer $j$, the production set $Y_{j}$ is convex and $0 \in Y_{j}$.

(4) For each $j$ and every $y_{j} \in Y_{j}$ associated with a weakly Pareto optimal consumption allocation, $Y_{j}$ is $\omega$-proper at $y_{j}$ in the following sense adapted from Tourky (1999): There exist in $L$ a convex set $\widehat{Y}_{j}\left(y_{j}\right)$ and a convex set $Z_{j}\left(y_{j}\right)$ such that

(a) the vector $y_{j}-\omega$ is a $\tau$-interior point of $\widehat{Y}_{j}\left(y_{j}\right)$,

(b) $\widehat{Y}_{j}\left(y_{j}\right) \cap Z_{j}\left(y_{j}\right)=Y_{j}$, and

(c) $0 \in Z_{j}\left(y_{j}\right)$ and $Z_{j}\left(y_{j}\right)-L_{+} \subseteq Z_{j}\left(y_{j}\right)$,

(d) for every $u>0$ and every couple $\left\{z_{j}, z_{j}^{\prime}\right\}$ of elements of $Z_{j}\left(y_{j}\right)$, if $z_{j} \leq u$ and $z_{j}^{\prime} \leq u$, then there exists $z \in Z_{j}\left(y_{j}\right)$ such that $z_{j} \leq z \leq u$ and $z_{j}^{\prime} \leq z \leq u$.

An economy satisfying these assumptions will be referred to as an $\omega$-proper economy.

Compactness: For some Hausdorff linear topology $\sigma$ on $L$, the set $X_{\omega}$ of all consumption allocations is $\sigma^{m}$-compact and preferences have $\sigma$-open (in $X_{i}$ ) lower sections $P_{i}^{-1}\left(x_{i}\right)=$ $\left\{x_{i}^{\prime} \in X_{i}: x_{i} \in P_{i}\left(x_{i}^{\prime}\right)\right\}$.

It is worth noticing that in the consumption case if $L$ is a Riesz space, then our assumptions on the sets $Z_{i}\left(x_{i}\right)$ are satisfied by the assumption of Tourky (1998) where each $Z_{i}\left(x_{i}\right)$ is a convex lattice containing $0, \omega_{i}, x_{i}$ and satisfying $Z_{i}\left(x_{i}\right)+L_{+} \subseteq Z_{i}\left(x_{i}\right)$. Our assumptions however do not imply that $L_{+}$is a lattice cone. For the production case, the sets $Z_{j}\left(y_{j}\right)$ correspond to the pretechnology sets defined by Mas-Colell (1986b) and Richard (1989).

The following lemma follows from the previous assumptions and Florenzano (1990).

\footnotetext{
${ }^{3}$ The ideas in this definition go back to Debreu and Scarf (1963). An important reference is also Aubin (1979). Edgeworth equilibria were introduced and studied in Aliprantis, Brown, and Burkinshaw (1987a).
} 
Lemma 3.4. In our economy, Edgeworth equilibria exist and belong to the fuzzy core.

As seen in Allouch and Florenzano (2004), one can prove that if preference correspondences are derived from quasiconcave utility functions defined on general consumption sets, our compactness condition can be replaced by a weaker compactness assumption made in the utility space on the "utility set". ${ }^{4}$

\section{Decentralizing Edgeworth equilibria}

For the discussion of this section, we fix an Edgeworth equilibrium consumption allocation $\left(\bar{x}_{i}\right)_{i \in I}$, thus a fuzzy core consumption allocation of $\mathcal{E}$, and $\left(\bar{y}_{j}\right)_{j \in J}$, the associated production allocation. Such an allocation $\left(\left(\bar{x}_{i}\right)_{i \in I},\left(\bar{y}_{j}\right)_{j \in J}\right)$ exists by Lemma 3.4. The proof of its possible decentralization by prices in $L^{\prime}$ will adapt ideas of (Florenzano, 2003, Chapter 5.3.4) where is proved the decentralization of Edgeworth equilibria of an $\omega$-proper production economy defined on a vector lattice commodity space. For technical details not covered in this paper, we refer to that chapter.

Since $L_{+}$is by (A1) generating, we can choose $u>0$ in $L$ such that the order interval $[-u, u]$ contains $\bar{x}_{i}, \omega_{i}, \bar{y}_{j}$ for all $i \in I$ and $j \in J$. Now consider the ordered vector subspace $L_{u}=$ $\bigcup_{\lambda>0} \lambda[-u, u]$ equipped with the order topology. This space is Archimedean (a property inherited from $L$ of which the positive cone is $\tau$-closed) and has $u$ as an order unit. ${ }^{5}$ Its order topology (i.e., the finest locally convex topology on $L_{u}$ for which every order interval is bounded) is normable [see (Schaefer, 1971, Chapter V 6.2)]. More precisely, the gauge $\|\cdot\|_{u}$ of $[-u, u]$, defined for each $z \in L_{u}$ by

$$
\|z\|_{u}=\inf \{\lambda>0: \quad-\lambda u \leq z \leq \lambda u\},
$$

is a norm on $L_{u}$ that generates the order topology whose closed unit ball is precisely the order interval $[-u, u]$ and $u$ is an interior point of $L_{u}^{+}=L_{u} \cap L_{+}$. Moreover, it follows from (A2) (namely from the fact that $[-u, u]$ is $\tau$-bounded) that on $L_{u}$ the order topology is finer than the topology induced by $\tau$. In addition, it is not difficult to see that the cone $L_{u}^{+}$is $\|\cdot\|_{u}$-closed in $L_{u}{ }^{6}{ }^{6}$

Let $\mathcal{E}_{u}$ be the economy $\mathcal{E}$ restricted to $L_{u}$ in an obvious way. For each consumer $i, X_{i}^{u}=X_{i} \cap L_{u}$, and for each $x_{i} \in X_{i}^{u}, P_{i}^{u}\left(x_{i}\right)=P_{i}\left(x_{i}\right) \cap L_{u}$; for each producer $j, Y_{j}^{u}=Y_{j} \cap L_{u}$. It is easily seen that the consumption allocation $\left(\bar{x}_{i}\right)_{i \in I}$ is an Edgeworth equilibrium and belongs to the fuzzy core of $\mathcal{E}_{u}$. It follows from the $\omega$-properness of $\mathcal{E}$ that for each $i$ and any $0<\alpha \leq 1$, the vector $\bar{x}_{i}+\alpha \omega$ belongs to the norm-interior of $P_{i}^{u}\left(\bar{x}_{i}\right)$. But then from the standard decentralization result in presence of an interiority property that there exists a nonzero $\bar{p}_{u} \in\left(L_{u},\|\cdot\|_{u}\right)^{\prime}$ such that $\left(\bar{x}, \bar{y}, \bar{p}_{u}\right)$ is a quasi-equilibrium of the restricted economy $\mathcal{E}_{u}$ and $\bar{p}_{u}(\omega)>0$.

Before going further, let us recall an extension lemma due to Podczeck (1996) whose a proof can be found in (Florenzano, 2003, Lemma 5.3.1, p. 134).

Lemma 4.1 (Podczeck). Let $(L, \tau)$ be an ordered topological vector space, let $K$ be a vector subspace of $L$ (endowed with the induced order), let $A$ be a convex subset of $K$ such that $A+K_{+} \subseteq A$, let

\footnotetext{
${ }^{4}$ That is, the set of feasible and individually rational utility vectors.

${ }^{5}$ That is, the order interval $[-u, u]$ is radial at the origin.

${ }^{6}$ Indeed, if $\left\{x_{n}\right\} \subseteq L_{+}$satisfies $-\frac{1}{n} u \leq x-x_{n} \leq \frac{1}{n} u$ for all $n$, then from $x=\left(x-x_{n}\right)+x_{n} \geq x-x_{n} \geq-\frac{1}{n} u$ and the Archimedean property we easily obtain $x \geq 0$.
} 
$V$ be a convex $\tau$-open subset of $L$ such that $V \cap A \neq \varnothing$, and let $a \in A \cap \operatorname{cl} V$. If $p$ is a linear functional on $K$ satisfying

$$
p \cdot a \leq p \cdot x, \text { for all } x \in V \cap A,
$$

then there exists some $\pi \in L^{\prime}$ such that $\pi_{\mid K} \leq p$, and

$$
p \cdot(a-x)=\pi \cdot(a-x) \text { for each } x \in A \text { with } x \leq a .
$$

The following obvious consequence of the preceding lemma was stated in Florenzano and Marakulin (2001).

Corollary 4.2. Let $(L, \tau)$ be an ordered topological vector space, let $K$ be a vector subspace of $L$ (endowed with the induced order), let $A$ be a convex subset of $K$ such that $A-K_{+} \subseteq A$, let $V$ be a convex $\tau$-open subset of $L$ such that $V \cap A \neq \varnothing$, and let $a \in A \cap \operatorname{cl} V$. If $p$ is a linear functional on $K$ satisfying

$$
p \cdot a \geq p \cdot x, \text { for all } y \in V \cap A,
$$

then there exists some $\pi \in L^{\prime}$ such that $\pi_{\mid K} \leq p$, and

$$
p \cdot(a-y)=\pi \cdot(a-y) \text { for each } y \geq a, y \in A .
$$

Our next step associates with the quasi-equilibrium price $\bar{p}_{u}$ a finite list of $\tau$-continuous linear functionals $\pi^{u}=\left(\left(\pi_{i}^{u}\right)_{i \in I},\left(\pi_{j}^{u}\right)_{j \in J}\right)$ defined on the whole commodity space. These continuous linear functionals can be thought of as personalized supporting prices for each consumer and each producer at the corresponding component of the allocation $(\bar{x}, \bar{y})$.

Proposition 4.1. In our economy, there exist $\left(\pi_{i}^{u}\right)_{i \in I},\left(\pi_{j}^{u}\right)_{j \in J}$ in $L^{\prime}$ such that:

(1) For each consumer $i$ we have

(a) $\pi_{i}^{u} \leq \bar{p}_{u}$ on $L_{u}$,

(b) $\pi_{i}^{u} \cdot \widehat{P}_{i}\left(\bar{x}_{i}\right) \geq \pi_{i}^{u} \cdot \bar{x}_{i}$, and

(c) if $z_{i} \in Z_{i}\left(\bar{x}_{i}\right) \cap L_{u}$ satisfies $z_{i} \leq \bar{x}_{i}$, then

$$
\bar{p}_{u} \cdot\left(\bar{x}_{i}-z_{i}\right)=\pi_{i}^{u} \cdot\left(\bar{x}_{i}-z_{i}\right)=\mathcal{R}_{\pi^{u}}\left(\bar{x}_{i}-z_{i}\right) .
$$

(2) For each producer $j$ we have

(a) $\pi_{j}^{u} \leq \bar{p}_{u}$ on $L_{u}$,

(b) $\pi_{j}^{u} \cdot \widehat{Y}_{j}\left(\bar{y}_{j}\right) \leq \pi_{j}^{u} \cdot \bar{y}_{j}$, and

(c) if $z_{j} \in Z_{j}\left(\bar{y}_{j}\right) \cap L_{u}$ satisfies $z_{j} \geq \bar{y}_{j}$, then

$$
\bar{p}_{u} \cdot\left(z_{j}-\bar{y}_{j}\right)=\pi_{j}^{u} \cdot\left(z_{j}-\bar{y}_{j}\right)=\mathcal{R}_{\pi^{u}}\left(z_{j}-\bar{y}_{j}\right) .
$$

(3) Moreover,

(a) $\mathcal{R}_{\pi^{u}} \leq \bar{p}_{u}$ on $L_{u}$, and

(b) if $v \leq \omega$ satisfies $v=\sum_{i \in I} v_{i}-\sum_{j \in J} w_{j} \in\left[\sum_{i \in I}\left(Z_{i}\left(\bar{x}_{i}\right) \cap L_{u}\right)-\sum_{j \in J}\left(Z_{j}\left(\bar{y}_{j}\right) \cap L_{u}\right)\right]$, then

$$
\bar{p}_{u}(\omega-v)=\mathcal{R}_{\pi^{u}}(\omega-v) .
$$

Proof. Using Lemma 4.1 and its corollary with $K=L_{u}$ and for each $i, a=\bar{x}_{i}, A=Z_{i}\left(\bar{x}_{i}\right) \cap L_{u}$, $V=\operatorname{int} \widehat{P}_{i}\left(\bar{x}_{i}\right)$, for each $j, a=\bar{y}_{j}, A=Z_{j}\left(\bar{y}_{j}\right) \cap L_{u}, V=\operatorname{int} \widehat{Y}_{j}\left(\bar{y}_{j}\right)$, the existence of $\left(\pi_{i}^{u}\right)_{i \in I},\left(\pi_{j}^{u}\right)_{j \in J}$ in $L^{\prime}$ satisfying (1), (2) and (3)(a) is a straightforward consequence of the fact that $\left(\bar{x}, \bar{y}, \bar{p}_{u}\right)$ is 
a quasi-equilibrium of the restricted economy $\mathcal{E}_{u}$ and of the $\omega$-properness assumptions at each component of the allocation $(\bar{x}, \bar{y})$.

To prove (3)(b), assume that $v \leq \omega, v=\sum_{i \in I} v_{i}-\sum_{j \in J} w_{j}$ with each $v_{i} \in Z_{i}\left(\bar{x}_{i}\right) \cap L_{u}$ and each $w_{j} \in Z_{j}\left(\bar{y}_{j}\right) \cap L_{u}$. Let $z_{i} \in Z_{i}\left(\bar{x}_{i}\right) \cap L_{u}$ be such that $z_{i} \leq\left\{v_{i}, \bar{x}_{i}\right\}$ and $z_{j} \in Z_{j}\left(y_{j}\right) \cap L_{u}$ be such that $z_{j} \geq\left\{w_{j}, \bar{y}_{j}\right\}$. Such $z_{i}$ and $z_{j}$ exist in view of the definition of $L_{u}$ and our assumptions on sets $Z_{i}\left(\bar{x}_{i}\right)$ and $Z_{j}\left(\bar{y}_{j}\right)$. On one hand, using $(1)(\mathrm{c}),(2)(\mathrm{c})$ and the superadditivity of $\mathcal{R}_{\pi^{u}}$, it follows from (1)(a) and (2)(a) that

$$
\begin{aligned}
\left(\bar{p}_{u}-\mathcal{R}_{\pi^{u}}\right)(\omega-v) & =\left(\bar{p}_{u}-\mathcal{R}_{\pi^{u}}\right)\left(\sum_{i \in I} \bar{x}_{i}-\sum_{j \in J} \bar{y}_{j}-\sum_{i \in I} v_{i}+\sum_{j \in J} w_{j}\right) \\
& \leq\left(\bar{p}_{u}-\mathcal{R}_{\pi^{u}}\right)\left(\sum_{i \in I}\left(\bar{x}_{i}-z_{i}\right)+\sum_{j \in J}\left(z_{j}-\bar{y}_{j}\right)\right)=0 .
\end{aligned}
$$

On the other hand, it follows also from (1)(a) and (2)(a) that $\left(\bar{p}_{u}-\mathcal{R}_{\pi^{u}}\right)(\omega-v) \geq 0$, which implies $\left(\bar{p}_{u}-\mathcal{R}_{\pi^{u}}\right)(\omega-v)=0$

The following corollary can be seen as an analogue for the production economy $\mathcal{E}_{u}$ of Proposition 5.1 in Aliprantis et al. (2004b) which extends at several instances statement (1) of Theorem 7.5 in Aliprantis et al. (2001) .

Corollary 4.3. We have in addition:

(1) $x_{i} \in P_{i}^{u}\left(\bar{x}_{i}\right) \Longrightarrow \mathcal{R}_{\pi_{u}}\left(x_{i}-z_{i}\right) \geq \mathcal{R}_{\pi_{u}}\left(\bar{x}_{i}-z_{i}\right)$ for every $z_{i} \in Z_{i}\left(\bar{x}_{i}\right) \cap L_{u}, z_{i} \leq\left\{x_{i}, \bar{x}_{i}\right\}$.

(2) $y_{j} \in Y_{j}^{u} \Longrightarrow \mathcal{R}_{\pi_{u}}\left(z_{j}-y_{j}\right) \geq \mathcal{R}_{\pi_{u}}\left(z_{j}-\bar{y}_{j}\right)$ for every $z_{j} \in Z_{j}\left(\bar{y}_{j}\right) \cap L_{u}, z_{j} \geq\left\{y_{j}, \bar{y}_{j}\right\}$.

(3) If for each $i, z_{i}^{\prime} \in Z_{i}\left(\bar{x}_{i}\right) \cap L_{u}, z_{i}^{\prime} \leq\left\{\omega_{i}, \bar{x}_{i}\right\}$, if for each $j, z_{j}^{\prime} \in Z_{j}\left(\bar{y}_{j}\right) \cap L_{u}, z_{j}^{\prime} \geq\left\{0, \bar{y}_{j}\right\}$, then

(a) $\mathcal{R}_{\pi_{u}}\left(\omega-\sum_{i \in I} z_{i}^{\prime}+\sum_{j \in J} z_{j}^{\prime}\right)=\sum_{i \in I} \pi_{i}^{u}\left(\bar{x}_{i}-z_{i}^{\prime}\right)+\sum_{j \in J} \pi_{j}^{u}\left(z_{j}^{\prime}-\bar{y}_{j}\right)$, and

(b) for each $i, \pi_{i}^{u} \cdot\left(\bar{x}_{i}-z_{i}^{\prime}\right)+\sum_{j \in J} \theta_{i j} \pi_{j}^{u} \cdot\left(z_{j}^{\prime}-\bar{y}_{j}\right) \geq \mathcal{R}_{\pi_{u}}\left(\omega_{i}-z_{i}^{\prime}+\sum_{j \in J} \theta_{i j} z_{j}^{\prime}\right)$.

Proof. To prove (1), let us assume $x_{i} \in P_{i}^{u}\left(\bar{x}_{i}\right)$ and $z_{i} \in Z_{i}\left(\bar{x}_{i}\right) \cap L_{u}, z_{i} \leq\left\{x_{i}, \bar{x}_{i}\right\}$. Recall that $P_{i}\left(\bar{x}_{i}\right)=\widehat{P}_{i}\left(\bar{x}_{i}\right) \cap Z_{i}\left(\bar{x}_{i}\right)$, thus that $P_{i}^{u}\left(\bar{x}_{i}\right)=\widehat{P}_{i}\left(\bar{x}_{i}\right) \cap Z_{i}\left(\bar{x}_{i}\right) \cap L_{u}$. We then easily deduce from Proposition 4.1 that:

$$
\mathcal{R}_{\pi_{u}}\left(x_{i}-z_{i}\right) \geq \pi_{i}^{u} \cdot\left(x_{i}-z_{i}\right) \geq \pi_{i}^{u} \cdot\left(\bar{x}_{i}-z_{i}\right)=\mathcal{R}_{\pi_{u}}\left(\bar{x}_{i}-z_{i}\right) .
$$

The proof of (2) is done symmetrically. The proof of (3) goes as follows.

Using the superadditivity of $\mathcal{R}_{\pi_{u}}$, the last assertion of Proposition 4.1, and assuming that for each $i, z_{i}^{\prime} \in Z_{i}\left(\bar{x}_{i}\right) \cap L_{u}, z_{i}^{\prime} \leq\left\{\omega_{i}, \bar{x}_{i}\right\}$, and for each $j, z_{j}^{\prime} \in Z_{j}\left(\bar{y}_{j}\right) \cap L_{u}, z_{j}^{\prime} \geq\left\{0, \bar{y}_{j}\right\}$, we get

$$
\begin{gathered}
\bar{p}_{u}\left(\omega-\sum_{i \in I} z_{i}^{\prime}+\sum_{j \in J} z_{j}^{\prime}\right)=\mathcal{R}_{\pi_{u}}\left(\omega-\sum_{i \in I} z_{i}^{\prime}+\sum_{j \in J} z_{j}^{\prime}\right) \geq \sum_{i \in I} \mathcal{R}_{\pi^{u}}\left(\bar{x}_{i}-z_{i}^{\prime}\right)+\sum_{j \in J} \mathcal{R}_{\pi^{u}}\left(z_{j}^{\prime}-\bar{y}_{j}\right) \\
=\sum_{i \in I} \pi_{i}^{u}\left(\bar{x}_{i}-z_{i}^{\prime}\right)+\sum_{j \in J} \pi_{j}^{u}\left(z_{j}^{\prime}-\bar{y}_{j}\right)=\bar{p}_{u}\left(\omega-\sum_{i \in I} z_{i}^{\prime}+\sum_{j \in J} z_{j}^{\prime}\right) .
\end{gathered}
$$

This proves the first assertion of $(3)$.

Finally, recall that $\left(\bar{x}, \bar{y}, \bar{p}_{u}\right)$ is a quasi-equilibrium of $\mathcal{E}_{u}$. We thus have for every $i$,

$$
\bar{p}_{u} \cdot \bar{x}_{i}=\bar{p}_{u} \cdot \omega_{i}+\sum_{j \in J} \theta_{i j} \bar{p}_{u} \cdot \bar{y}_{j} .
$$


From (4.1), using (1)(c) and (2)(c) of Proposition 4.1, we deduce easily from that for each $i$,

$$
\begin{gathered}
\mathcal{R}_{\pi_{u}}\left(\bar{x}_{i}-z_{i}^{\prime}\right)+\sum_{j \in J} \theta_{i j} \mathcal{R}_{\pi_{u}}\left(z_{j}^{\prime}-\bar{y}_{j}\right)=\bar{p}_{u} \cdot\left(\bar{x}_{i}-z_{i}^{\prime}\right)+\sum_{j \in J} \theta_{i j} \bar{p}_{u} \cdot\left(z_{j}^{\prime}-\bar{y}_{j}\right) \\
=\bar{p}_{u} \cdot\left(\omega_{i}-z_{i}^{\prime}\right)+\sum_{j \in J} \theta_{i j} \bar{p}_{u} \cdot z_{j}^{\prime} \geq \mathcal{R}_{\pi_{u}}\left(\omega_{i}-z_{i}^{\prime}+\sum_{j \in J} \theta_{i j} z_{j}^{\prime}\right)
\end{gathered}
$$

which completes the proof.

Let us now recall some notions of properness for functions on $L_{+}$.

Definition 4.4. Let $v \in L_{+}$be such that $v>0$. We say that a function $f: L_{+} \rightarrow \mathbb{R}$ is:

(1) $v$-proper at some $x \in L_{+}$, if there exists a convex set $F$ such that:

(a) $x+v$ is an interior point of $F$, and

(b) $F \cap L_{+}=\left\{y \in L_{+}: f(y)>f(x)\right\}$.

(2) v-pointwise proper at $x \in L_{+}$, if there exists an open pointed convex cone $\Gamma_{x}$ such that:

(a) $-v \in \Gamma_{x}$, and

(b) $\left(x-\Gamma_{x}\right) \cap\left\{y \in L_{+}: f(y)>f(x)\right\}=\varnothing$.

Considering the set $\left\{y \in L_{+}: f(y)>f(x)\right\}$ as a preferred set for a preference correspondence defined on $L_{+}$by the utility function $f$, the reader recognizes in these definitions the usual notions of $v$-pointwise properness as defined by Mas-Colell (1986a) and of $v$-properness as defined by Tourky (1998). Mas-Colell (1986a) defines uniform properness on a subset $X$ of $L_{+}$as properness at every $x \in X$ with a properness vector and a properness cone independent of $x .^{7}$ The definition of uniform $v$-properness given here is quite similar.

Definition 4.5. A function $f: L_{+} \rightarrow \mathbb{R}$ is uniformly v-proper on $X \subset L_{+}$if for every $x \in X$ there exist a convex set $F_{x}$ and a $\tau$-neighborhood $V$ of 0 (independent of $x$ ) such that

(a) $x+v+V \subseteq F_{x}$, and

(b) $F_{x} \cap L_{+}=\left\{y \in L_{+}: f(y)>f(x)\right\}$.

We now introduce the following additional assumption of compatibility between the order structure and the topology $\tau$ of the commodity space of our economy.

(A3) For any finite list $f=\left(f_{1}, \ldots, f_{\ell}\right)$ of continuous linear functionals satisfying $f_{k}(\omega)>0$ for each $k$, the Riesz-Kantorovich functional $\mathcal{R}_{f}$ is uniformly $\omega$-proper at any point $\omega^{\prime} \geq \omega$.

Remark 4.6. Condition (A3) is automatically satisfied if the commodity space is a locally convex vector lattice and its dual is a vector sublattice of the order dual. Indeed, in this case, it follows from Theorem 2.1 that the Riesz-Kantorovich functionals are linear and $\tau$-continuous. Assume now that $f$ is a finite list of continuous linear functionals $\left(f_{k}\right)_{k=1}^{K}$ such that $f_{k}(\omega)>0$ for each $k$ (which implies $\mathcal{R}_{f}(\omega)>0$ ). For each $\omega^{\prime} \in L_{+}$, define $F_{\omega^{\prime}}=\left\{y \in L: g(y)>g\left(\omega^{\prime}\right)\right\}$, where $g$ is the continuous linear functional which coincides with $\mathcal{R}_{f}$ on $L_{+}$. If $V$ is a $\tau$-neighborhood of 0 such that $g(\omega+V)>0$, then $g\left(\omega^{\prime}+\omega+V\right)>g\left(\omega^{\prime}\right)$, thus $\omega^{\prime}+\omega+V \subseteq F_{\omega^{\prime}}$, while $F_{\omega^{\prime}} \cap L_{+}=\{y \in$ $\left.L: \mathcal{R}_{f}(y)>\mathcal{R}_{f}\left(\omega^{\prime}\right)\right\}$.

${ }^{7}$ If $v$ is the properness vector, recall that Mas-Colell's uniform properness on $L_{+}$implies $v$-properness [see Tourky (1998)]. 
We can provide a simple way of identifying order structures on Banach spaces that satisfy condition (A3). Recall that a subset $A$ of an ordered set dominates a point $x$, in symbols $A \geq x$, if $a \geq x$ holds true for each $a \in A$.

Theorem 4.7. Let $L$ be an ordered Banach space with a closed generating cone, weakly compact order intervals and admitting a strictly positive linear functional. In this case, condition (A3) is satisfied if for any finite list $f=\left(f_{1}, \ldots, f_{\ell}\right)$ of positive (necessarily continuous) linear functionals there exists a weak $k^{*}$ compact set $G \subseteq L^{\prime}$ that dominates each $f_{k}$ and satisfies

$$
\inf \left\{g(x): g \in L^{\prime} \text { and } g \geq f_{k} \text { for all } k=1, \ldots, \ell\right\}=\min \{g(x): g \in G\} \text {, }
$$

for each $x \in L_{+}$.

Proof. Let $f=\left(f_{1}, \ldots, f_{\ell}\right)$ be a finite list of continuous linear functionals satisfying $f_{k}(\omega)>0$ for all $k$. Since in ordered normed spaces the norm boundedness of order intervals is equivalent to the normality of the cone, it follows that $L_{+}^{\prime}$ is a generating cone. In particular, there exists some positive linear functional $h$ such that $h \geq-f_{k}$ for each $k$. Now if $h_{1}$ is a strictly positive linear functional and we let $p=h+h_{1}$, then $f_{k}+p$ is strictly positive for each $k=1, \ldots, \ell$. By our assumption there exists a weak* compact set $G \subseteq L^{\prime}$ that dominates each $f_{k}+p$ such that

$$
\inf \left\{g(x): g \in L^{\prime} \text { and } g \geq f_{k}+p \text { for all } k=1, \ldots, \ell\right\}=\min \{g(x): g \in G\},
$$

holds true for each $x \in L_{+}$.

Let $\mathcal{W}=\{x \in L: g(x) \geq 0$ for all $g \in G\}$. Notice that $\mathcal{W}$ is a closed wedge that contains $\omega$ as an interior point. Moreover, we have $L_{+} \subseteq \mathcal{W}$. Next, define the mapping $\mathcal{M}: \mathcal{W} \rightarrow \mathbb{R}$ via the formula

$$
\mathcal{M}(x)=\min \{g(x): g \in G\} .
$$

We show that $\mathcal{M}$ is a norm lower semicontinuous function. Suppose that $\left\|x_{\alpha}-x\right\| \rightarrow 0$, where $\left\{x_{\alpha}\right\}$ is a net of $\mathcal{W}$ such that $\mathcal{M}\left(x_{\alpha}\right) \leq c$. Let $\left\{g_{\alpha}\right\}$ be the net of corresponding minimizers in $G$. By passing to a subnet (if necessary), we can assume without loss of generality that $g_{\alpha} \stackrel{w^{*}}{\rightarrow} g$ in $L^{\prime}$. It easily follows that $g_{\alpha}\left(x_{\alpha}\right) \rightarrow g(x)$. Therefore, $\mathcal{M}(x) \leq g(x) \leq c$.

Clearly, $\mathcal{M}$ is positively homogeneous. That is $\mathcal{M}(\lambda x)=\lambda \mathcal{M}(x)$ for all $\lambda \geq 0$. We show that it is super-additive. Pick $x, y \in \mathcal{W}$ and pick $h_{1}, h_{2} \in G$ such that let $h_{1}(x)=\mathcal{M}(x)$ and $h_{2}(y)=\mathcal{M}(y)$ be the respective minimizers. Now for any $g \in G$ we have $g(x+y) \geq h_{1}(x)+h_{2}(y)$. Therefore, $\mathcal{M}(x+y) \geq \mathcal{M}(x)+\mathcal{M}(y)$.

We return to the list $f$. Let $M_{f}(x)=\min \{g(x): g \in G-p\}$. Notice that for any $x \in \mathcal{W}$ we have $M_{f}(x)=\mathcal{M}(x)-p(x)$. Therefore, for $x, y \in \mathcal{W}$ we have

$$
M_{f}(x+y)=\mathcal{M}(x+y)-p(x+y) \geq \mathcal{M}(x)+\mathcal{M}(y)-p(x)-p(y)=M_{f}(x)+M_{f}(y) .
$$

That is, $M_{f}$ is super-additive. Clearly, it is also positively homogeneous. These two facts imply that the upper sections $U_{x}=\left\{y \in \mathcal{W}: M_{f}(y) \geq M_{f}(x)\right\}$ are convex sets for each $x \in \mathcal{W}$.

Now we know that $M_{f}(\omega)=\mathcal{M}(\omega)-p(\omega)>0$. Since $\mathcal{M}$ is lower semicontinuous, $M_{f}$ is likewise lower semicontinuous and so there exists an open ball $V$ centered at $\omega$ satisfying $V \subseteq \mathcal{W}$ and $\mathcal{M}(v)>p(v)$ for all $v \in V$.

Next, pick any $x \in \mathcal{W}$ and notice that for any $v \in V$ we have $M_{f}(x+v) \geq M_{f}(x)+M_{f}(v)$. Because $M_{f}(v)=\mathcal{M}(v)-p(v)$, this implies that $M_{f}(x+v)>M_{f}(x)$. Therefore, $M_{f}$ is $\omega$-uniformly proper at each $x \in L_{+}$.

It is shown in (Aliprantis and Tourky, 2002, Theorem 7.6, p. 2072) that for any $x \in L_{+}$that if $\pi=\left(f_{1}+p, f_{2}+p, \ldots, f_{\ell}+p\right)$, then we have $\mathcal{R}_{\pi}(x)=\mathcal{M}(x)$. Clearly, $\mathcal{R}_{f}=\mathcal{R}_{\pi}-p$. Therefore, $\mathcal{R}_{f}(x)=M_{f}(x)$ for all $x \in L_{+}$, and the proof is finished. 
Examples of closed generating cones that satisfy the assumptions of Theorem 4.7 are the generating polyhedral cones in finite dimensional spaces.

We move to the first application of (A3).

Proposition 4.2. Under (A3), there exists a price system $\bar{\pi}_{u} \in L^{\prime}$ such that $\bar{\pi}_{u} \cdot \omega>0$ and $\left(\bar{x}, \bar{y}, \bar{\pi}_{u}\right)$ is a quasi-equilibrium of $\mathcal{E}_{u}$.

Proof. Let us consider the set $\Omega$ of all $\omega^{\prime}=\omega-\sum_{i \in I} z_{i}^{\prime}+\sum_{j \in J} z_{j}^{\prime}$, where for each $i, z_{i}^{\prime} \in Z_{i}\left(\bar{x}_{i}\right) \cap L_{u}$, $z_{i}^{\prime} \leq\left\{0, \omega_{i}, \bar{x}_{i}\right\}$, and for each $j, z_{j}^{\prime} \in Z_{j}\left(\bar{y}_{j}\right) \cap L_{u}, z_{j}^{\prime} \geq\left\{0, \bar{y}_{j}\right\}$. This set is nonempty in view of the definition of $L_{u}$ and our assumptions on $Z_{i}\left(\bar{x}_{i}\right)$ and $Z_{j}\left(\bar{y}_{j}\right)$. It is directed by the relation

$$
\omega^{\prime \prime} \geq \omega^{\prime} \text { if and only if for each } i \text { and } j, z_{i}^{\prime \prime} \leq z_{i}^{\prime} \text { and } z_{j}^{\prime \prime} \geq z_{j}^{\prime} .
$$

Moreover, if $\omega^{\prime} \in \Omega$ then $z_{i}^{\prime} \leq 0$ for each $i$ and $z_{j}^{\prime} \geq 0$ for each $j$ imply $\omega^{\prime} \geq \omega>0$. From (3) in Corollary 4.3, it follows that the Riesz-Kantorovich functional $\mathcal{R}_{\pi_{u}}$ is exact at each $\omega^{\prime} \in \Omega$. From Proposition 4.1, it is easily deduced that for each $i$, either $\pi_{i}^{u}=0$ or $\pi_{i}^{u} \cdot \omega>0$, that for each $j$, either $\pi_{j}^{u}=0$ or $\pi_{j}^{u} \cdot \omega>0$, that $\mathcal{R}_{\pi^{u}}(\omega)=\bar{p}_{u}(\omega)>0$, thus that at least one of the $\pi_{i}^{u}$, $\pi_{j}^{u}$ is nonzero. Set $P\left(\omega^{\prime}\right)=\left\{z \in L_{+}: \mathcal{R}_{\pi_{u}}(z)>\mathcal{R}_{\pi_{u}}\left(\omega^{\prime}\right)\right\}$. Applying Assumption (A3), there exist a 0-neighborhood $V$ and for each $\omega^{\prime} \in \Omega$, a convex set $\widehat{P}\left(\omega^{\prime}\right)$ such that $\omega^{\prime}+\omega+V \subseteq \widehat{P}\left(\omega^{\prime}\right)$ and $P\left(\omega^{\prime}\right)=L_{+} \cap \widehat{P}\left(\omega^{\prime}\right)$.

Note that

$$
\mathcal{R}_{\pi_{u}}\left(\omega^{\prime}+\alpha \omega\right) \geq \mathcal{R}_{\pi_{u}}\left(\omega^{\prime}\right)+\alpha \mathcal{R}_{\pi_{u}}(\omega)>\mathcal{R}_{\pi_{u}}\left(\omega^{\prime}\right) \text { every } 0<\alpha \leq 1,
$$

so that $\omega^{\prime}$ belongs to the closure of $\widehat{P}\left(\omega^{\prime}\right)$. Now, if $z \in \widehat{P}\left(\omega^{\prime}\right) \cap L_{u}^{+}$, then $\mathcal{R}_{\pi_{u}}(z)>\mathcal{R}_{\pi_{u}}\left(\omega^{\prime}\right)=\bar{p}_{u}\left(\omega^{\prime}\right)$. From $\mathcal{R}_{\pi^{u}} \leq \bar{p}_{u}$ on $L_{u}$, it follows that $\bar{p}_{u} \cdot z>\bar{p}_{u} \cdot \omega^{\prime}$. If we define $\left.X=\left\{z \in L_{u}^{+}: \bar{p}_{u} \cdot z \leq \bar{p}_{u} \cdot \omega^{\prime}\right)\right\}$, the last observation can be rephrased as $X \cap \widehat{P}\left(\omega^{\prime}\right)=\varnothing$, so that to each $\omega^{\prime} \in \Omega$, we can associate a nonzero $\bar{\pi}_{\omega \prime} \in L^{\prime}$ which separates $\widehat{P}\left(\omega^{\prime}\right)$ and $X$, that is,

$$
\bar{\pi}_{\omega^{\prime}} \cdot X \leq \bar{\pi}_{\omega^{\prime}} \cdot \omega^{\prime} \leq \bar{\pi}_{\omega^{\prime}} \cdot \widehat{P}\left(\omega^{\prime}\right) .
$$

Since $\omega^{\prime}+\omega$ is an interior point of $\widehat{P}\left(\omega^{\prime}\right)$, we have $\bar{\pi}_{\omega^{\prime}} \cdot \omega>0$, and we can normalize prices letting

$$
\bar{p}_{u} \cdot \omega=\mathcal{R}_{\pi^{u}}(\omega)=\bar{\pi}_{\omega^{\prime}} \cdot \omega=1 .
$$

Let $L_{\omega^{\prime}}$ be the ordered vector subspace $L_{\omega^{\prime}}=\bigcup_{\lambda>0} \lambda\left[-\omega^{\prime}, \omega^{\prime}\right]$. Clearly, $L_{\omega^{\prime}} \subset L_{u}$. We first claim that $\bar{\pi}_{\omega^{\prime}}=\bar{p}_{u}$ on $L_{\omega^{\prime}}$. Indeed, for every $z \in L_{u}^{+}$, we know that

$$
\bar{p}_{u} \cdot z \leq \bar{p}_{u} \cdot \omega^{\prime} \Longrightarrow \bar{\pi}_{\omega^{\prime}} \cdot z \leq \bar{\pi}_{\omega^{\prime}} \cdot \omega^{\prime} .
$$

So, using the existence of Lagrange multipliers for a convex programming problem, ${ }^{8}$ there exist two real numbers $\lambda_{1} \geq 0$ and $\lambda_{2} \geq 0$ not all equal to zero such that $\lambda_{1}\left[\bar{\pi}_{\omega^{\prime}} \cdot z-\bar{\pi}_{\omega^{\prime}} \cdot \omega^{\prime}\right] \leq \lambda_{2}\left[\bar{p}_{u} \cdot z-\bar{p}_{u} \cdot \omega^{\prime}\right]$ for every $z \in L_{u}^{+}$. From $\bar{\pi}_{\omega^{\prime}} \cdot \omega=\bar{p}_{u} \cdot \omega>0$, we easily deduce that $\left(\lambda_{1}, \lambda_{2}\right) \gg 0$. Letting successively $z=0$ and $z=2 \omega^{\prime}$, we also see that for some $\lambda>0, \bar{\pi}_{\omega^{\prime}} \cdot \omega^{\prime}=\lambda \bar{p}_{u} \cdot \omega^{\prime}$ and $\bar{\pi}_{\omega^{\prime}} \cdot z \leq \lambda \bar{p}_{u} \cdot z$ for every $z \in L_{u}^{+}$. The previous inequality holds in particular for every $z \in L_{\omega^{\prime}}^{+}$. Since $\omega^{\prime}$ is an interior point (for the order topology) of $L_{\omega^{\prime}}^{+}$, it follows that $\bar{\pi}_{\omega^{\prime}}=\lambda \bar{p}_{u}$ on $L_{\omega^{\prime}}$. Recalling that $\bar{\pi}_{\omega^{\prime}} \cdot \omega=\bar{p}_{u} \cdot \omega$, it follows that $\lambda=1$.

\footnotetext{
${ }^{8} \mathrm{~A}$ simple proof of the existence of Lagrange multipliers for a convex programming problem can be found in (Barbu, and Precupanu, 1986, Chapter 3, Theorem 1.1). See also Fan et al. (1957) and (Aliprantis and Border, 2006, Chapter 5, Theorem 5.70).
} 
Recalling that for each $i$, we have $\bar{p}_{u} \cdot \bar{x}_{i}=\bar{p}_{u} \cdot \omega_{i}+\sum_{j \in J} \theta_{i j} \bar{p}_{u} \cdot \bar{y}_{j}$, that is,

$$
\bar{p}_{u} \cdot\left(\bar{x}_{i}-z_{i}^{\prime}\right)+\sum_{j \in J} \theta_{i j} \bar{p}_{u} \cdot\left(z_{j}^{\prime}-\bar{y}_{j}\right)=\bar{p}_{u} \cdot\left(\omega_{i}-z_{i}^{\prime}\right)+\sum_{j \in J} \theta_{i j} \bar{p}_{u} \cdot z_{j}^{\prime},
$$

we deduce from our first claim:

$$
\bar{\pi}_{\omega^{\prime}} \cdot\left(\bar{x}_{i}-z_{i}^{\prime}\right)+\sum_{j \in J} \theta_{i j} \bar{\pi}_{\omega^{\prime}} \cdot\left(z_{j}^{\prime}-\bar{y}_{j}\right)=\bar{\pi}_{\omega^{\prime}} \cdot\left(\omega_{i}-z_{i}^{\prime}\right)+\sum_{j \in J} \theta_{i j} \bar{\pi}_{\omega^{\prime}} \cdot z_{j}^{\prime},
$$

and, thus, for each $i$ and for every $\omega^{\prime} \in \Omega$,

$$
\bar{\pi}_{\omega^{\prime}} \cdot \bar{x}_{i}=\bar{\pi}_{\omega^{\prime}} \cdot \omega_{i}+\sum_{j \in J} \theta_{i j} \bar{\pi}_{\omega^{\prime}} \cdot \bar{y}_{j} .
$$

We next claim that for some $\mu>0$ (depending on $\left.\omega^{\prime}\right), \mu \bar{\pi}_{\omega^{\prime}}$ is a supergradient of $\mathcal{R}_{\pi_{u}}$ at $\omega^{\prime}$. Indeed, from $\mathcal{R}_{\pi_{u}}(z) \geq \mathcal{R}_{\pi_{u}}\left(\omega^{\prime}\right) \Longrightarrow \bar{\pi}_{\omega^{\prime}}(z) \geq \bar{\pi}_{\omega^{\prime}}\left(\omega^{\prime}\right)$ for every $z \in L_{+}$, one deduces, as previously, the existence of two real numbers $\mu_{1} \geq 0$ and $\mu_{2} \geq 0$ not all equal to zero such that $\mu_{1}\left[\bar{\pi}_{\omega^{\prime}} \cdot z-\bar{\pi}_{\omega^{\prime}} \cdot \omega^{\prime}\right] \geq \mu_{2}\left[\mathcal{R}_{\pi_{u}}(z)-\mathcal{R}_{\pi_{u}}\left(\omega^{\prime}\right)\right]$ for every $z \in L_{+}$. As previously, from $\mathcal{R}_{\pi_{u}}(\omega)=\bar{\pi}_{\omega^{\prime}} \cdot \omega>$ 0 , one deduces $\left(\mu_{1}, \mu_{2}\right) \gg 0$, so that for some $\mu>0, \mu \bar{\pi}_{\omega^{\prime}} \cdot \omega^{\prime}=\mathcal{R}_{\pi_{u}}\left(\omega^{\prime}\right)$ and $\mu \bar{\pi}_{\omega^{\prime}} \cdot z \geq \mathcal{R}_{\pi_{u}}(z)$ for every $z \in L_{+}$, which proves the claim.

Fix now $(x, y) \in \prod_{i \in I} P_{i}^{u}\left(\bar{x}_{i}\right) \times \prod_{j \in J} Y_{j}^{u}$. Let $\omega_{0}^{\prime}=\omega-\sum_{i \in I} z_{i 0}^{\prime}+\sum_{j \in J} z_{j_{0}}^{\prime}$ where for each $i$, $z_{i 0}^{\prime} \in Z_{i}\left(\bar{x}_{i}\right) \cap L_{u}, z_{i 0}^{\prime} \leq\left\{0, x_{i}, \omega_{i}, \bar{x}_{i}\right\}$, and for each $j, z_{j_{0}}^{\prime} \in Z_{j}\left(\bar{y}_{j}\right) \cap L_{u}, z_{j_{0}}^{\prime} \geq\left\{y_{j}, 0, \bar{y}_{j}\right\}$. Once again, this is possible in view of the definition of $L_{u}$ and our assumptions on $Z_{i}\left(\bar{x}_{i}\right)$ and $Z_{j}\left(\bar{y}_{j}\right)$. For every $\omega^{\prime} \geq \omega_{0}^{\prime}$ in $\Omega$, applying Theorem 2.3 and using (1) and (2) of Proposition 4.1, we get:

$$
x_{i} \in P_{i}^{u}\left(\bar{x}_{i}\right) \Longrightarrow \bar{\pi}_{\omega^{\prime}} \cdot\left(x_{i}-z_{i}^{\prime}\right) \geq \bar{\pi}_{\omega^{\prime}} \cdot\left(\bar{x}_{i}-z_{i}^{\prime}\right) \Longrightarrow \bar{\pi}_{\omega^{\prime}} \cdot x_{i} \geq \bar{\pi}_{\omega^{\prime}} \cdot \bar{x}_{i}
$$

and for each $i, \pi_{i}^{u}\left(\bar{x}_{i}-z_{i}^{\prime}\right)=\bar{\pi}_{\omega^{\prime}} \cdot\left(\bar{x}_{i}-z_{i}^{\prime}\right)$,

$$
y_{j} \in Y_{j}^{u} \Longrightarrow \bar{\pi}_{\omega^{\prime}} \cdot\left(z_{j}^{\prime}-y_{j}\right) \geq \bar{\pi}_{\omega^{\prime}} \cdot\left(z_{j}^{\prime}-\bar{y}_{j}\right) \Longrightarrow \bar{\pi}_{\omega^{\prime}} \cdot y_{j} \leq \bar{\pi}_{\omega^{\prime}} \cdot \bar{y}_{j}
$$

and for each $j, \pi_{j}^{u}\left(z_{j}^{\prime}-\bar{y}_{j}\right)=\bar{\pi}_{\omega^{\prime}} \cdot\left(z_{j}^{\prime}-\bar{y}_{j}\right)$.

Let $V$ be the $\tau$-neighborhood of 0 referred to in Assumption A3. We can assume that $V$ is convex and circled. From (4.2), we deduce that $\bar{\pi}_{\omega^{\prime}} \cdot V \leq \bar{\pi}_{\omega^{\prime}} \cdot \omega=1$, thus that each $\bar{\pi}_{\omega^{\prime}}$ belongs to $V^{0}$, the polar set of $V$ in $L^{\prime}$. Since $L$ is locally convex, it follows from Alaoglu-Bourbaki's theorem that $V^{0}$ is $\tau$-equicontinuous, thus $\sigma\left(L^{\prime}, L\right)$-compact. Passing to a subnet if necessary, we can assume that $\bar{\pi}_{\omega^{\prime}} \stackrel{\sigma\left(L^{\prime}, L\right)}{\longrightarrow} \bar{\pi}_{u} \in L^{\prime}$ such that $\bar{\pi}_{u} \cdot \omega=1$. Passing to limit in the relations (4.5), (4.6) and (4.4), we get $\bar{\pi}_{u} \cdot x_{i} \geq \bar{\pi}_{u} \bar{x}_{i}, \bar{\pi}_{u} \cdot y_{j} \leq \bar{\pi}_{u} \bar{y}_{j}$, and for each $i, \bar{\pi}_{u} \cdot \bar{x}_{i}=\bar{\pi}_{u} \cdot \omega_{i}+\sum_{j \in J} \theta_{i j} \bar{\pi}_{u} \cdot \bar{y}_{j}$, which completes the proof that $\left(\bar{x}, \bar{y}, \bar{\pi}_{u}\right)$ is a quasi-equilibrium of $\mathcal{E}_{u}$.

To go further, we now consider the family $\mathcal{U}$ of all $u>0$ in $L$ such that the order interval $[-u, u]$ contains $\bar{x}_{i}, \omega_{i}, \bar{y}_{j}$ for all $i \in I$ and $j \in J$ and notice that $\mathcal{U}$ is a directed set. We will apply the previous result to the economies $\mathcal{E}_{u}$ defined as above and will pass to limit.

The next proposition is the main result of this paper.

Proposition 4.3. Under (A3), there exists a price system $\bar{\pi} \in L^{\prime}$ such that $\bar{\pi} \cdot \omega>0$ and $(\bar{x}, \bar{y}, \bar{\pi})$ is a quasi-equilibrium of $\mathcal{E}$. This quasi-equilibrium is non-trivial if for some $\lambda>0$, $\lambda \omega \in \omega+\sum_{j \in J} Y_{j}-\sum_{i \in I} X_{i}$. 
Proof. For each $u \in \mathcal{U}$, in view of the previous proposition and of its proof, let $\bar{\pi}_{u} \in L^{\prime}$ such that $\bar{\pi}_{u} \cdot \omega^{\prime} \leq \bar{\pi}_{u} \cdot \widehat{P}\left(\omega^{\prime}\right)$ and $\left(\bar{x}, \bar{y}, \bar{\pi}_{u}\right)$ is a quasi-equilibrium of $\mathcal{E}_{u}$. Let $V$ be the convex and circled $\tau$-neighborhood of 0 such that $\omega^{\prime}+\omega+V \subseteq \widehat{P}\left(\omega^{\prime}\right)$. As previously, $\bar{\pi}_{u} \cdot V \leq \bar{\pi}_{u} \cdot \omega=1$, and passing to a subnet if necessary, we can assume that $\bar{\pi}_{u} \stackrel{\sigma\left(L^{\prime}, L\right)}{\longrightarrow} \bar{\pi} \in L^{\prime}$ such that $\bar{\pi} \cdot \omega=1$.

We now claim that $\bar{\pi}$ supports the allocation $(\bar{x}, \bar{y})$. To see that, fix now $(x, y) \in \prod_{i \in I} P_{i}\left(\bar{x}_{i}\right) \times$ $\prod_{j \in J} Y_{j}$. By construction of $\mathcal{U}$, all $x_{i}, y_{j}$ belong to some $L_{u_{0}}$ for $u_{0} \in \mathcal{U}$ and consequently to any $L_{u} \supset L_{u_{0}}$. Passing to limit in the relations $\bar{\pi}_{u} \cdot x_{i} \geq \bar{\pi}_{u} \bar{x}_{i}$ and $\bar{\pi}_{u} \cdot y_{j} \leq \bar{\pi}_{u} \bar{y}_{j}$, we get $\bar{\pi} \cdot x_{i} \geq \overline{\pi x}_{i}$ and $\bar{\pi} \cdot y_{j} \leq \overline{\pi y}_{j}$.

Passing to limit in the relations $\bar{\pi}_{u} \cdot \bar{x}_{i}=\bar{\pi}_{u} \cdot \omega_{i}+\sum_{j \in J} \theta_{i j} \bar{\pi}_{u} \cdot \bar{y}_{j}$, we get that for each $i$, $\bar{\pi} \cdot \bar{x}_{i}=\bar{\pi} \cdot \omega_{i}+\sum_{j \in J} \theta_{i j} \bar{\pi} \cdot \bar{y}_{j}$, proving that $(\bar{x}, \bar{y}, \bar{\pi})$ is a quasi-equilibrium of $\mathcal{E}$.

Finally, assume that for some $\lambda>0, \lambda \omega \in \omega+\sum_{j \in J} Y_{j}-\sum_{i \in I} X_{i}$. From $\bar{\pi} \cdot \omega=1$, we deduce that there exist $x \in \prod_{i \in I} X_{i}, y \in \prod_{j \in J} Y_{j}$ such that $\bar{\pi} \cdot\left(\omega+\sum_{j \in J} y_{j}-\sum_{i \in I} x_{i}\right)>0$. We then have for some $i_{0}$,

$$
\bar{\pi} \cdot x_{i_{0}}<\bar{\pi} \cdot \omega_{i_{0}}+\sum_{j \in J} \theta_{i_{0} j} \bar{\pi} \cdot y_{j} \leq \bar{\pi} \cdot \omega_{i_{0}}+\sum_{j \in J} \theta_{i_{0} j} \bar{\pi} \cdot \bar{y}_{j}=\bar{\pi} \cdot \bar{x}_{i_{O}},
$$

which proves that the quasi-equilibrium is non-trivial.

Remark 4.8. The condition for non-triviality is in particular satisfied if inaction is possible as well for consumers $\left(0 \in X_{i}\right)$ as for producers $\left(0 \in Y_{j}\right)$.

We conclude the paper with our main result.

Theorem 4.9. If (A1), (A2), and (A3) hold true for the commodity space, then an w-proper and compact production economy has a non-trivial quasi-equilibrium.

\section{REFERENCES}

C. D. Aliprantis and K. C. Border, Infinite Dimensional Analysis: A Hitchhiker's Guide, $3^{\text {rd }}$ Edition, Springer-Verlag, Heidelberg and New York, 2006.

C. D. Aliprantis, D. J. Brown, and O. Burkinshaw, Edgeworth equilibria. Econometrica 55(3): 1109-1137, 1987a.

C. D. Aliprantis, D. J. Brown, and Burkinshaw, Edgeworth equilibria in production economies. J. Econom. Theory 43(3):252-291, 1987b.

C. D. Aliprantis, D. J. Brown, and J. Werner, Minimum-cost portfolio insurance. J. Econom. Dynam. Control 24(3):1703-1719, 2000.

C. D. Aliprantis and O. Burkinshaw, Positive Operators, Academic Press, New York and London, 1985.

C. D. Aliprantis and O. Burkinshaw, Locally Solid Riesz Spaces with Applications to Economics, American Mathematical Society, Mathematical Surveys and Monographs, Volume 105, Providence, RI, 2003.

C. D. Aliprantis, M. Florenzano, F. Martins Da Rocha, and R. Tourky, Equilibrium analysis in financial markets with countably many securities. J. Math. Econom. 40(3):683-699, $2004 a$.

C. D. Aliprantis, M. Florenzano, and R. Tourky, General equilibrium analysis in ordered topological vector spaces. J. Math. Econom. 40(3):247-269, 2004 b.

C. D. Aliprantis, M. Florenzano, and R. Tourky. Linear and non-linear price decentralization. J. Econom. Theory 121(3):51-74, 2005. 
C. D. Aliprantis, P. K. Monteiro, and R. Tourky, Non-marketed options, non-existence of equilibria, and non-linear prices. J. Econom. Theory 114(3):345-357, 2004c.

C. D. Aliprantis and R. Tourky, The super order dual of an ordered vector space and the RieszKantorovich formula. Trans.. Amer. Math. Soc. 354(3):2055-2077, 2002.

C. D. Aliprantis, R. Tourky, and N. C. Yannelis, The Riesz-Kantorovich formula and general equilibrium theory. J. Math. Econom. 34(3):55-76, 2000.

C. D. Aliprantis, R. Tourky, and N. C. Yannelis, A theory of value: equilibrium analysis beyond vector lattices. J. Econom. Theory 100(3):22-72, 2001.

N. Allouch and M. Florenzano, Edgeworth and Walras equilibria of an arbitrage-free exchange economy. Econom. Theory 23(3):353-370, 2004.

J. P. Aubin, Mathematical Methods of Game and Economic Theory, North-Holland, Amsterdam and New York, 1979.

V. Barbu and T. Precupanu, Convexity and Optimization in Banach spaces, D. Reidel Publishing Company, Boston and Lancaster, 1986.

G. Debreu and H. Scarf, A limit theorem on the core of an economy. Internat. Econom. Rev. 4 (3):235-246, 1963.

K. Fan, I. L. Glicksberg, and A. J. Hoffman, Systems of inequalities involving convex functions. Proc. Amer. Math. Soc. 13(3):617-622, 1957.

M. Florenzano, Edgeworth equilibria, fuzzy core and equilibria of a production economy without ordered preferences. J. Math. Anal. Appl. 153(3):18-36, (1990).

M. Florenzano, General Equilibrium Analysis: Existence and Optimality Properties of Equilibria, Kluwer Academic Publishers, Boston and London, 2003.

M. Florenzano and V. Marakulin, Production equilibria in vector lattices. Econom. Theory 17(3): 577-598, 2001.

P. J. Laurent, Approximation et Optimisation, Herman, Paris, 1972.

A. Mas-Colell, The price equilibrium existence problem in topological vector lattices. Econometrica 54(3):1039-1055, 1986a.

A. Mas-Colell, Valuation equilibria and Pareto optimum revisited. In W. Hildenbrand and A. Mas-Colell (eds.), Contributions to Mathematical Economics. In honor of Gérard Debreu, NorthHolland, Amsterdam, 317-331, 1986.

A. Mas-Colell and S. F. Richard, A new approach to the existence of equilibria in vector lattices. J. Econom. Theory 53(3):1-11, 1991.

J. J. Moreau, Fonctionnelles convexes, Séminaire sur les équations aux dérivées partielles, Collège de France, 1966-1967.

K. Podczeck, Equilibria in vector lattices without ordered preferences or uniform properness. $J$. Math. Econom. 25(3):465-485, 1996.

S. F. Richard, A new approach to production equilibria in vector lattices. J. Math. Econom. 18 (3):41-56, 1989.

R. T. Rockafellar and R. J.-B. Wets, Variational Analysis, Springer-Verlag, Heildelberg and New York, 1998

H. H. Schaefer, Topological Vector Spaces, Springer Verlag, New York and Heildelberg, 1971.

R. Tourky, A new approach to the limit theorem on the core of an economy in vector lattices. $J$. Econom. Theory 78(3):321-328, 1998.

R. Tourky, The limit theorem on the core of a production economy in vector lattices with unordered preferences. Econom. Theory 14(3):219-226, 1999. 
W. R. Zame, Competitive equilibria in production economies with an infinite dimensional space. Econometrica, 55(3):1075-1108, 1987. 